\title{
La productividad científica argentina en Ciencias Sociales: Economía, Psicología, Sociología y Ciencia Política en el CONICET (2004-2008)
}

\author{
Ernesto R. Gantman*
}

Resumen: Este trabajo analiza una base de datos elaborada por el autor a partir de los curriculum vitae de 414 investigadores argentinos en economía, sociología, ciencia política y psicología con afiliación institucional en el Consejo Nacional de Investigaciones Científicas y Técnicas (CONICET). Se examinan los patrones de publicación de dichos investigadores, revelándose diferentes patrones por disciplina científica. Además, se presenta un análisis de regresión multivariado, cuyos resultados indican que el lugar de formación doctoral de los investigadores está asociado en forma positiva y estadísticamente significativa al circuito de revistas científicas en las que los mismos publican.

Palabras clave: productividad científica, Ciencias Sociales, Argentina, países en desarrollo.

\section{Scientific productivity of Social Sciences in Argentina: Economics, Psychology, Sociology, and Political Science in the CONICET (2004-2008)}

\begin{abstract}
This study analyses a dataset elaborated by the author, using as sources the CVs of 414 Argentine researchers in economics, sociology, political science, and psychology affiliated with the Consejo Nacional de Investigaciones Cientificas y Técnicas (CONICET). The findings indicate that the researchers' publication patterns vary across the different scientific disciplines. Moreover, a multivariate regression analysis reveals that the place where the researchers' doctoral studies occurred has a positive and statistically significant association with the circuit of scholarly journals in which they choose to publish.
\end{abstract}

Keywords: scientific productivity, social sciences, Argentina, developing countries.

\section{Introducción}

Es una creencia ampliamente aceptada que las ciencias sociales tienen características idiosincrásicas distintivas de acuerdo a su origen nacional o regional. No suele hablarse de una química alemana como diferente a una química italiana o estadounidense, pero sí es posible pensar en una sociología francesa, una sociología anglo-americana o una sociología latinoamericana (Sztompka, 2009). Más

* Universidad de Buenos Aires. Universidad de Belgrano. Argentina. Correo-e: ernesto.gantman@ comunidad.ub.edu.ar.

Recibido: 27-12-2010; 2. ${ }^{\text {a }}$ versión: 09-05-2011; aceptado; 11-05-2011. 
allá de eventuales diferencias en contenido, marcos teóricos o enfoques metodológicos, las características nacionales de la producción en ciencias sociales tienen un correlato en diferentes hábitos de publicación por parte de los científicos que las practican. Así, Hicks (2004) en su distinción entre distintas literaturas en las ciencias sociales señala que hay un corpus internacional y un corpus local de publicaciones en revistas científicas con referato. En este sentido, el hecho de que la producción de los científicos sociales que no se desempeñan en instituciones del mundo anglosajón no se vea adecuadamente representada en las bases de datos internacionales que recopilan información sobre la producción en revistas científicas genera un problema (Narvaez-Berthelemot y Russell, 2001; Sancho, 1992; Sanz et al. 2002; Van Leeuwen et al., 2001). Las bases internacionales no resultan, entonces, muy útiles a la hora de evaluar la producción de los científicos sociales que no pertenecen al mundo anglosajón. El análisis bibliométrico de la cobertura de publicaciones científicas en bases internacionales como el Social Sciences Citation Index (SSCI) demuestra que este problema resulta especialmente grave en las ciencias sociales (Archambault et al, 2006; Archambault y Larivière, 2010; Kahn, 2010). Este sesgo de la cobertura de publicaciones locales implica la invisibilidad de los investigadores en ciencias sociales que trabajan en países en desarrollo. En el caso de los mismos, la ausencia de visibilidad puede deberse a diversos motivos: baja calidad de las publicaciones científicas locales o problemas propios de la administración de dichas revistas, como la falta de fondos para sostener una periodicidad de aparición adecuada (Vessuri, 1995).

Si la producción en ciencias sociales nacionales de países no anglosajones no está adecuadamente reflejada en lo que Shenhav y Kamens (1991) han denominado el núcleo institucionalizado de la producción científica, resulta importante cuantificar, en casos particulares, cuál es el nivel de participación en la literatura internacional (supuestamente de superior calidad) frente al grado de participación en la literatura local. Sin embargo, los estudios descriptivos de los patrones de publicaciones de investigadores de países en desarrollo (por ej., Molteni y Zulueta, 2002; Sanz Casado y Conforti, 2005) son bastante escasos. Por ello, en este trabajo se intenta responder a esta cuestión a través del análisis empírico, utilizando datos de curriculum vitae de investigadores en ciencias sociales de un país en desarrollo, la República Argentina. Dicha estrategia metodológica permite obtener más información que la usualmente disponible en las bases científicas internacionales (Gaillard, 1992; Dietz y otros, 2003). Por otra parte, esta fuente de recolección de datos permite, adicionalmente, abordar otro interrogante relacionado: ¿qué factores explican la elección geográfica de revistas científicas por parte de los investigadores?

\section{Material y metodos}

A partir de información proveniente del sitio web del Consejo Nacional de Investigaciones Científicas y Técnicas (CONICET) de la República Argentina se obtuvieron datos de las publicaciones realizadas por 414 investigadores afiliados 
a dicha institución: 199 de la disciplina sociología (clasificación KS5), 76 de economía (clasificación KS6), 57 de psicología (subdisciplina 5300 y desagregadas) y 83 de ciencia política y relaciones internaciones (subdisciplina 5400 y desagregadas). Esta información surge de los curriculum vitae resumidos de los investigadores, recopilados de la citada fuente (www.conicet.gov.ar) en febrero y marzo de 2011. Se escogieron dichas áreas porque se considera que pueden tener un importante grado de internacionalización (entendida como presencia en revistas internacionales) y, por tanto, ser más relevantes para comparar la orientación de la producción en términos de publicaciones locales vs. internacionales (Russell y Ainsworth, 2010). En cambio, no se recopilaron datos de áreas disciplinarias como historia, ya que ésta tiene una clara orientación al conocimiento local y, consecuentemente, podría ser esperable una reducida participación a nivel internacional. En el caso de sociología, el área incluye también a demografía, pero se las considera áreas de conocimiento afines y se presume que tienen perfiles muy similares, por lo cual la agregación de las mismas no constituye un problema. En el caso de economía, el área considerada contiene otras dos disciplinas, ya que se trata de economía, ciencias de la gestión y administración pública, pero la proporción de investigadores pertenecientes a dichas disciplinas es mínima. Naturalmente, existe cierto nivel de arbitrariedad en la asignación o, más precisamente, la auto asignación de los investigadores a áreas disciplinarias, ya que sus áreas de actuación en ocasiones son multidisciplinarias (por ej.: sociólogos que publican en revistas de historia, expertos en administración pública que figuran en el área de ciencias políticas, etc.), pero se ha preferido respetar el criterio de demarcación disciplinaria del CONICET.

Los datos recopilados corresponden a la totalidad de investigadores cuya información sobre publicaciones figuraba en la base de datos de acceso público del CONICET, abarcando científicos sociales de todas las categorías de revista: asistente, adjunto, independiente, principal y superior. Al respecto, cabe señalar que la categorización de investigadores se realiza a partir de un proceso de evaluación interna y sólo aquellos con mérito suficiente son promocionados a una categoría más elevada. La antigüedad en la carrera de investigadores también es importante para el cambio de categoría, como surge del artículo 39 del estatuto que reglamenta la carrera de investigador científico del CONICET (Decreto Ley $20.464 / 73$, modificado por leyes 22.140 y 24.729). A los efectos comparativos, nuestro análisis desagrega la información sobre productividad según categoría de revista de los investigadores, consolidándose en una sola categoría las de principal y superior (que tienen un número muy reducido de miembros). En definitiva, esta clasificación marca una diferenciación en el nivel de antigüedad o experiencia de los investigadores, que podría tener influencia en su productividad.

Es pertinente realizar algunas precisiones sobre la representatividad de la muestra. Según las últimas cifras disponibles (datos oficiales a diciembre de 2008), el total de investigadores en ciencias sociales en la República Argentina era de 9989 (cifra que incluye tanto los que poseen dedicación exclusiva como parcial a dichas tareas). De acuerdo al tamaño total de la muestra y considerando un nivel 
de confianza de $95 \%$, se obtendrían resultados representativos con un margen de error de 4,71\%. No obstante, la representatividad debe ser calculada por disciplina, por las variaciones existentes entre las mismas. Para economía, con 1.411 investigadores a nivel país, se obtendrían resultados representativos con un intervalo de confianza de 10,94; sociología con 1.099 investigadores tiene un tamaño de muestra más representativo con un intervalo de confianza de 6,29, mientras que en psicología, con 1.170 investigadores, los resultados son significativos con un intervalo de 12,67. No hay datos para estimar la representatividad en el caso de la ciencia política. En cualquier caso, estos niveles de representatividad no son adecuados ya que la población de comparación es menor, debido a que hay un alto porcentaje de investigadores en ciencias sociales que se dedican en forma parcial a dichas tareas (Wainerman, 1999). Por otra parte, como los investigadores en este estudio no han surgido de un muestreo aleatorio, sino que forman parte de la carrera de investigador científico del CONICET, puede concluirse que la muestra está sesgada hacia los más productivos del sistema, además de restringirse a quienes tienen dedicación plena a la investigación. En consecuencia, las muestras no serían enteramente representativas del científico social argentino promedio, pero según su tamaño, podrían considerarse representativas de los más productivos del sistema.

Cabe señalar al respecto que los investigadores considerados en la muestra tienen, en la mayor parte de los casos, afiliación institucional en universidades argentinas públicas o privadas, pero su afiliación al CONICET garantiza que se trata de investigadores full-time. De hecho, la carrera de investigador científico en el CONICET tiene precisamente por objetivo garantizar dicha dedicación. Creado en 1958, para promover el desarrollo de la actividad científica en la República Argentina, el CONICET es una agencia pública que otorga becas y subsidios a investigadores, además de contar con un escalafón de investigadores científicos que disfrutan de ventajas como la seguridad de la dedicación full-time y una remuneración acorde a ella, mientras pertenezcan al mismo. Para acceder a integrar este cuerpo de investigadores, se atraviesa un estricto proceso de selección.

En encuestas realizadas entre miembros de la comunidad científica local, el CONICET figura como la entidad con mayor prestigio en el campo de la investigación académica (CONICET, 2006). Por otra parte, en términos de productividad, debe señalarse que los científicos afiliados al CONICET produjeron en 2004 un $70 \%$ del total de publicaciones científicas indexadas en SCI de autores afiliados a instituciones argentinas (CAICYT, 2005). Lo anterior sugiere que es razonable pensar que la muestra de científicos obtenida podría considerarse sesgada hacia los más productivos del sistema. Afirmación que se sustenta, además, en el hecho de que la evaluación para promoción en la carrera de investigadores del CONICET se basa en criterios de productividad tanto en calidad como en cantidad.

De la producción de los investigadores se relevaron los siguientes datos:

1. Publicaciones de artículos en revistas científicas, diferenciando según la localización geográfica de las revistas (locales, de otros países de Améri- 
ca Latina, españolas, francesas y resto) y el idioma de la publicación (de cualquier país pero publicadas en inglés, considerado lingua franca de la ciencia). En este último caso, además, se agregó una subcategoría: publicaciones en inglés indexadas en el SSCI (Social Sciences Citation Index), lo que responde a la intención de identificar los artículos publicados en revistas científicas que presumiblemente tienen mayor índice de impacto a nivel internacional (Jiménez Contreras, 1992; Giannoni, 2008; Morillo y otros, 1999).

2. Libros publicados como autor o editor, según lugar o idioma de publicación (publicados localmente, publicados en idioma inglés, publicados en países de América Latina o publicados en otros países en lenguas distintas al inglés).

3. Capítulos de libros, también clasificados según lugar e idioma de publicación.

Las reseñas bibliográficas publicadas en revistas con referato no se han considerado en el análisis. Los working papers también fueron excluidos del análisis.

A los efectos de verificar la integralidad de los datos suministrados en los curriculum vitae que figuran en el sitio web del CONICET, se cruzaron los mismos con datos de los respectivos investigadores obtenidos de otras fuentes (C.V. de sitios web de universidades, etc.). El período considerado en el análisis corresponde a la producción de los años 2004 a 2008. En algunos casos, los C.V. incluyen publicaciones anteriores, pero se eligió el período citado porque representa en forma mas completa la producción de los investigadores (en otros años, podrían registrarse omisiones). Para establecer las características de las publicaciones periódicas mencionadas en los C.V. de los investigadores, se recurrió a diversas fuentes, como los sitios web de las mismas y los listados de publicaciones suministrados por Thomson International.

Por último, se obtuvo información sobre las universidades en las cuales los investigadores completaron su doctorado. Como este dato no figuraba en los C.V. disponibles en el sitio del CONICET, se utilizaron otras fuentes de información como páginas de universidades o publicaciones de los propios investigadores.

El análisis descriptivo de datos contiene las medias por tipo de publicación y categoría de investigador, así como los promedios consolidados de todas las categorías. A los efectos de evitar distorsiones en la productividad media por investigador debidas a valores extremos en cada una de las disciplinas (científicos excepcionalmente productivos en los totales de algunas categorías), fueron excluidos de la muestra aquellos investigadores cuya productividad se ubicaba a mayor distancia que tres desvíos estándar de la media. El número de outliers fue de 13, quedando la muestra final compuesta por 401 investigadores.

También se presenta un análisis de regresión multivariado que toma como variables dependientes distintas categorías de publicaciones: artículos por lugar de publicación y libros locales. Como variables independientes se utilizan dummies según el país de obtención del doctorado (la categoría base de comparación 
es el doctorado local). Dada la naturaleza discreta de dichas variables dependientes, se ha utilizado un modelo de recuento de datos con una regresión binomial negativa. Los resultados se han obtenido mediante la utilización del software STATA 10.0 .

\section{Resultados}

En la tabla I se detalla la producción de los investigadores argentinos en economía en el período de referencia (2004-2008). Los datos corresponden a la producción media del período, por lo cual a los efectos de obtener medias anuales hay que dividir por cinco. Como puede observarse, en materia de artículos predomina la producción en revistas locales, mientras que la producción en revistas en inglés es la categoría que le sigue. Las publicaciones en revistas latinoamericanas tienen una media ligeramente inferior, que supera a la de las publicaciones en revistas españolas o francesas. La media de publicaciones en inglés en revistas indexadas en el SSCI $(0,70)$ corresponde a un $28,80 \%$ de las publicaciones locales y a un $14 \%$ del total de artículos. Cabe puntualizar que el SSCI indexa en ciencias sociales algunas revistas publicadas en otros idiomas, por lo cual la visibilidad de los investigadores argentinos en dicha base de datos es, en realidad, mayor. De hecho, la revista argentina Desarrollo Económico fue indexada durante el período de referencia (Luna-Morales y Collazo-Reyes, 2007). Calculando el índice de internacionalización como producción media en revistas internacionales sobre producción media total, obtenemos a nivel consolidado un valor de 51,40\%. La proporción de publicaciones a nivel regional (revistas locales más las de otros países de América Latina sobre total de publicaciones) asciende a $68 \%$.

La producción de libros tiene relevancia como modo de difundir las investigaciones de economistas argentinos, pero es inferior a la de artículos en revistas locales. La mayor parte de estos libros han sido publicados localmente, aunque se verifica también una mínima producción de libros en inglés. En materia de capítulos en libros, puede señalarse que el número promedio de capítulos en libros locales es levemente menor al número de artículos en revistas locales. Hay también producción de capítulos en libros publicados en el exterior.

Comparando la producción por categorías de investigador, se aprecia que, en general, las categorías superiores son más productivas en artículos en revistas locales y de países latinoamericanos así como en libros locales, aunque la relación no sea monótonamente creciente.

La producción en sociología se informa en la tabla II. El número de artículos en revistas locales es nuevamente la categoría predominante. En segundo lugar, aparecen los artículos publicados en revistas de América Latina. Las revistas en inglés ocupan el tercer lugar. A continuación, aparecen las publicaciones en revistas españolas y luego las revistas francesas. Es importante puntualizar, sin embargo, que la proporción de publicaciones en revistas en inglés indexadas en 


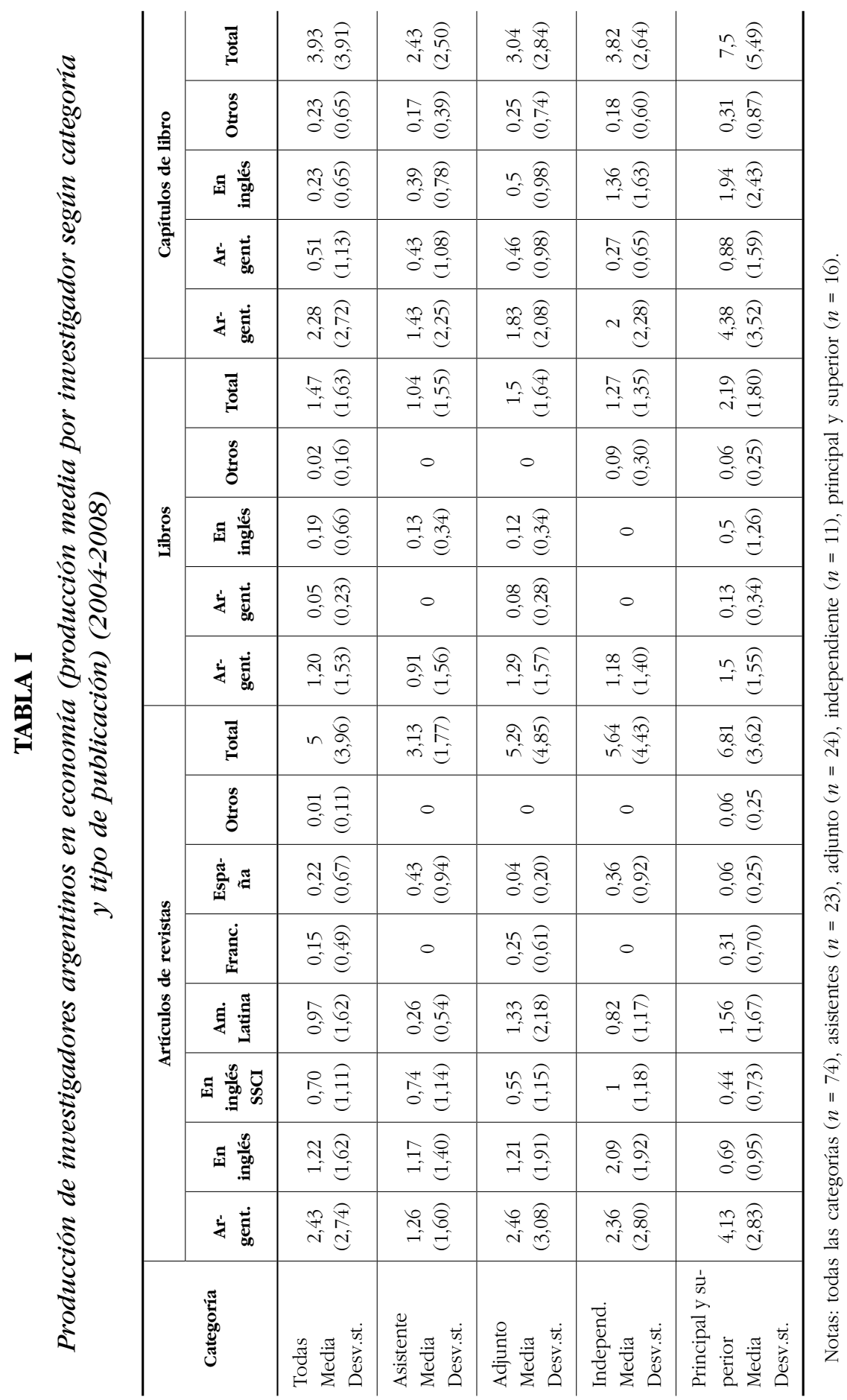




\begin{tabular}{|c|c|c|c|c|c|c|c|}
\hline \multirow{5}{*}{$\begin{array}{c}0 \\
0 \\
0 \\
8 \\
0 \\
0 \\
0 \\
5 \\
5 \\
0 \\
0 \\
0 \\
0 \\
0\end{array}$} & \multirow{5}{*}{ 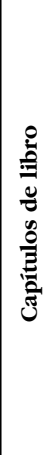 } & స్ّँّ & $\begin{array}{l}\stackrel{\widehat{n}}{n} \\
\forall+\hat{n}\end{array}$ & $\begin{array}{l}\approx \text { r } \\
\dot{r} \\
\dot{d}\end{array}$ & 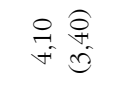 & $\begin{array}{l}\text { 을 } \\
\text { in } \\
\text { in }\end{array}$ & 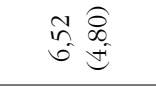 \\
\hline & & : & 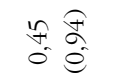 & $\begin{array}{l}\infty \\
\stackrel{\widehat{P}}{+} \\
0\end{array}$ & $\begin{array}{l}8 \approx \\
0 \\
0\end{array}$ & 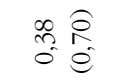 & $\begin{array}{l}\hat{\infty} \\
0_{0} \\
0\end{array}$ \\
\hline & & $\frac{\mathscr{B}}{g}$ & $\begin{array}{l}\text { से } \\
\hat{0}\end{array}$ & $\begin{array}{l}\text { t. } \\
\text { to } \\
0 \\
0\end{array}$ & $\begin{array}{l}\approx \\
\approx \\
0 \\
0\end{array}$ & 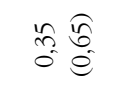 & \begin{tabular}{l}
$\infty$ \\
\multirow{2}{*}{} \\
$\dot{e}$
\end{tabular} \\
\hline & & $\dot{4}$ & $\begin{array}{l}\hat{b} \\
0 \stackrel{\vec{m}}{=}\end{array}$ & \begin{tabular}{ll}
\multirow{2}{*}{} & $\widehat{a}$ \\
0 & $=$
\end{tabular} & 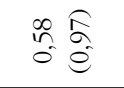 & $\begin{array}{l}\infty \\
\infty \\
\infty \\
0 \\
0\end{array}$ & 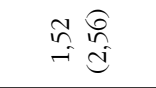 \\
\hline & & $\dot{4} \dot{4}$ & 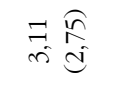 & $\begin{array}{l}a \\
\infty \\
i \\
i \\
i\end{array}$ & $\begin{array}{l}\text { ô } \\
\text { i } \\
\text { id }\end{array}$ & $+\frac{\hat{\alpha}}{\hat{d}}$ & $\begin{array}{l}0 \text { ह } \\
\text { ni } \\
0\end{array}$ \\
\hline 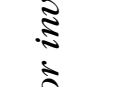 & \multirow{5}{*}{ : } & हुँ & $\begin{array}{l}m \\
\stackrel{n}{n} \\
\rightarrow=\end{array}$ & 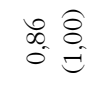 & 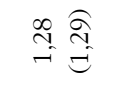 & 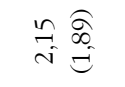 & 啇 \\
\hline$\frac{\pi}{2}$ & & $\stackrel{0}{0}$ & $\begin{array}{l}0 \\
0 \\
0 \\
0 \\
0\end{array}$ & $\begin{array}{l}\infty \text { E } \\
0 . \\
0 \\
0\end{array}$ & $\begin{array}{l}8 \\
0 \\
0 \\
0\end{array}$ & 0 & 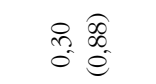 \\
\hline 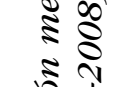 & & 畐 $\frac{\mathscr{8}}{\mathrm{g}}$ & $\begin{array}{l}\hat{I} \\
0 \\
0 \\
0\end{array}$ & $\begin{array}{l}\hat{2} \\
0 \\
0 \\
0\end{array}$ & $\begin{array}{l}\overrightarrow{0} \\
0 \\
0 \\
0\end{array}$ & $\begin{array}{l}\approx \\
: E \\
0 \\
0\end{array}$ & 0 \\
\hline 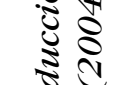 & & 安䓵 & 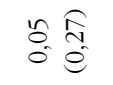 & $\begin{array}{l}\tilde{I} \\
0 \\
0 \\
0\end{array}$ & $\begin{array}{l}\text { t. } \\
0 \\
0 \\
0\end{array}$ & 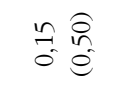 & 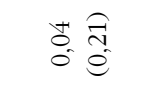 \\
\hline 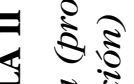 & & 安荜 & શે & 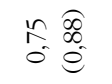 & 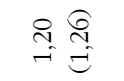 & 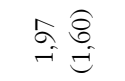 & 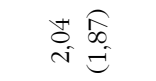 \\
\hline $\begin{array}{l}0 \\
0 \\
0 \\
0 \\
0 \\
0 \\
0\end{array}$ & \multirow{8}{*}{ 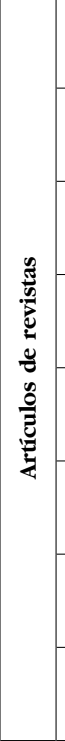 } & हुँ & 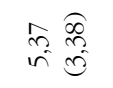 & 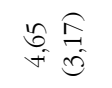 & $\begin{array}{l}\text { 누 } 0 \\
\text { in } \\
\text { in }\end{array}$ & 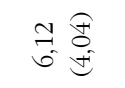 & 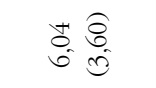 \\
\hline 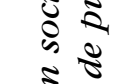 & & $\stackrel{0}{0}$ & $\begin{array}{l}\text { 角 } \\
\stackrel{0}{0}\end{array}$ & 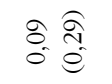 & 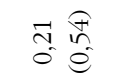 & $\begin{array}{l}\text { a } \widehat{\hat{\lambda}} \\
0 \text { है }\end{array}$ & 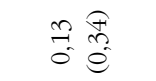 \\
\hline ڤ & & 善 & 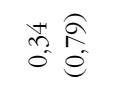 & \begin{tabular}{l}
$\approx$ \\
\multirow{2}{n}{} \\
0 \\
$\tilde{e}$
\end{tabular} & $\begin{array}{l}i \text { E } \\
\hat{0} \text { हे }\end{array}$ & 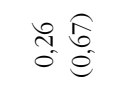 & 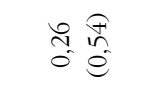 \\
\hline 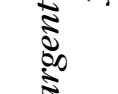 & & 节 & $\begin{array}{l}n \\
\hat{n} \\
0 \\
0\end{array}$ & 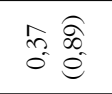 & 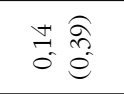 & 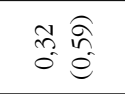 & 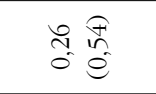 \\
\hline ङे & & 远蓄 & ì & $\begin{array}{l}\approx \approx \\
\approx \\
\approx\end{array}$ & 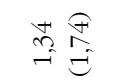 & 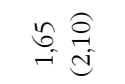 & $\begin{array}{l}\infty \\
\stackrel{\mathbb{I}}{\mathrm{I}} \\
\rightarrow-\mathbb{d}\end{array}$ \\
\hline$\frac{5}{5}$ & & 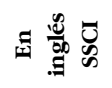 & 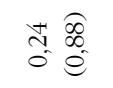 & $\begin{array}{l}\infty \\
0 . ~ \\
0 \\
0 \\
0\end{array}$ & 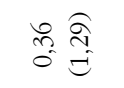 & $\begin{array}{l}\text { त् } \\
\text { E. } \\
0\end{array}$ & 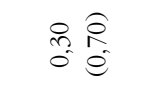 \\
\hline 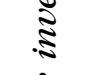 & & 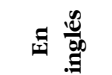 & $\begin{array}{l}\infty \\
\stackrel{\pi}{\pi} \\
0\end{array}$ & $\begin{array}{ll}\stackrel{o}{1} \\
\text { it }\end{array}$ & 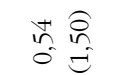 & $\begin{array}{l}\sqrt[H]{\vec{E}} \\
\hat{0}=\end{array}$ & $\begin{array}{l}\text { ते } \\
\hat{0}=\end{array}$ \\
\hline కิ & & 安若 & 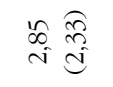 & $\begin{array}{l}\hat{A} \\
\text { i } \\
\text { id }\end{array}$ & $\underset{\substack{r \\
\text { i }}}{\stackrel{0}{d}}$ & $\begin{array}{l}8 \underset{+}{\mathbb{f}} \\
\dot{n}\end{array}$ & 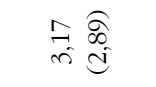 \\
\hline$\frac{5}{0}$ & & 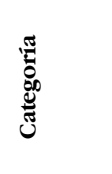 & 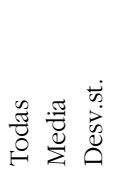 & 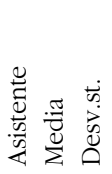 & 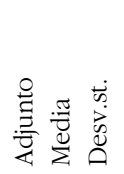 & 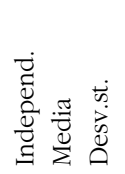 & 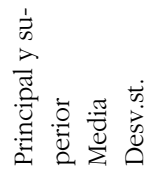 \\
\hline
\end{tabular}


el SSCI respecto a las publicaciones en revistas locales es bastante baja, apenas el $8,42 \%$ a nivel consolidado de categorías. El nivel de internacionalización asciende al 46,95\% de la producción. Sin embargo, dicha cifra está compuesta principalmente por las publicaciones en revistas latinoamericanas que corresponden a un $24 \%$ del total. Las publicaciones en inglés cubren un modesto 8,.94\% del total y sólo el 4,47\% corresponde a publicaciones en inglés indexadas en el SSCI.

Al igual que en el caso de los economistas, la producción de los sociólogos argentinos se vehiculiza de manera importante a través de libros, algo que ya se había puntualizado con relación a las ciencias sociales (Kishida y Matsui, 1997). A nivel consolidado, la relación entre libros publicados sobre artículos en revistas locales es de 0,45. Por otra parte, es importante señalar que los libros se publican principalmente a nivel nacional, representando las publicaciones internacionales cifras muy bajas. Los capítulos en libros publicados localmente constituyen la categoría de mayor producción de los sociólogos argentinos, pero su número no es demasiado superior al de los artículos en revistas nacionales, apenas 3,11 contra 2,85. El número de capítulos en libros publicados en otros países es bastante menor.

Analizando comparativamente la producción por categorías, vemos que las categorías superiores tienen, en general, mayor producción de artículos (aunque no en todas las categorías de artículos) y, particularmente, de libros publicados localmente. Esto último podría obedecer al mayor prestigio de los investigadores en la disciplina, lo cual les otorga un rol de gatekeepers dentro de la sociología local.

La tabla III presenta los resultados para los investigadores en ciencia política. Los mismos, en general, son bastante similares a los obtenidos en el caso de los sociólogos. En materia de artículos, predominan las publicaciones locales $(58,89 \%$ del total), registrándose una proporción muy baja de artículos en inglés indexados en el SSCI (2,75\% del total). A diferencia del caso de los sociólogos, el número de capítulos de libro $(3,18)$ es levemente inferior al de artículos en revistas locales $(3,27)$. Se observa también que el número de libros locales publicados aumenta en forma monótonamente creciente con la categoría de revista, lo que también se verifica en el caso de los sociólogos.

La producción de los investigadores en psicología muestra que la categoría de mayor producción corresponde a artículos en revistas locales (tabla IV). Sigue a continuación el número de artículos en revistas latinoamericanas y luego en revistas españolas. El número de capítulos en libros locales es bastante inferior al número de artículos en revistas locales (apenas el 37,8\% de este último). Los capítulos de libros no son un modo de difusión importante en esta disciplina, al menos en comparación con los artículos (el total de capítulos representa sólo el 26,35\% del total de artículos).

Se observa que el número promedio de artículos en revistas locales aumenta según la categoría de investigador, al igual que el número de artículos en revistas españolas. Aunque no en forma monótonamente creciente, también hay un aumento del número de capítulos de libros según la categoría y del número de libros 


\begin{tabular}{|c|c|c|c|c|c|c|c|}
\hline$\frac{8}{8}$ & \multirow{5}{*}{ 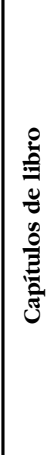 } & స్ & 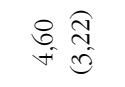 & 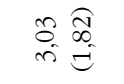 & 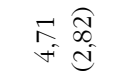 & 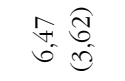 & $\begin{array}{l}\hat{n} \\
\hat{n} \\
\tilde{n}^{-\infty}\end{array}$ \\
\hline$\frac{2}{8}$ & & $\stackrel{\infty}{0}$ & $\begin{array}{l}\infty \\
\cdots \\
m \\
0 \\
0 \\
0\end{array}$ & $\begin{array}{l}\hat{\sigma} \\
\Rightarrow \tilde{\sigma} \\
\tilde{c}\end{array}$ & $\begin{array}{l}\tilde{Q} \\
\hat{n} \\
0\end{array}$ & $\begin{array}{l}: \\
0 \\
0\end{array}$ & $\begin{array}{l}\vec{\Xi} \\
\stackrel{\Xi}{\approx}\end{array}$ \\
\hline $\begin{array}{l}\overrightarrow{5} \\
0 \\
0 \\
0\end{array}$ & & 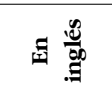 & 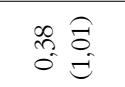 & $\begin{array}{l}\hat{A} \\
\Rightarrow \hat{n} \\
0\end{array}$ & $\begin{array}{l}\hat{\jmath} \\
\hat{\sigma} \\
\tilde{\sigma}\end{array}$ & 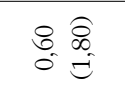 & $\begin{array}{l}\hat{A} \\
\stackrel{f}{\mathbb{Z}} \\
0\end{array}$ \\
\hline$\frac{5}{8}$ & & $\dot{4}$ & 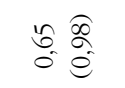 & 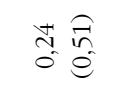 & $\begin{array}{l}\hat{x} \\
\hat{0}= \\
\tilde{\sigma}\end{array}$ & $\begin{array}{l}\infty \\
\infty \\
0 \\
0\end{array}$ & 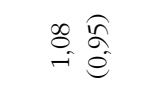 \\
\hline $\begin{array}{l}\tilde{S} \\
\vdots \\
\vdots\end{array}$ & & 安㗎 & 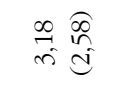 & $\begin{array}{l}\underset{*}{\mathbb{N}} \underset{i}{i} \\
\stackrel{i}{=}\end{array}$ & $\begin{array}{l}\text { ते } \widehat{E} \\
\dot{n} \mathbf{d}\end{array}$ & $\begin{array}{l}\stackrel{\leftrightarrow}{\vec{d}} \\
\stackrel{d}{d}\end{array}$ & $\begin{array}{l}\vec{m} \\
\vec{a} \\
\tilde{n}^{2}\end{array}$ \\
\hline 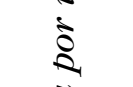 & \multirow{5}{*}{ : } & స్ & 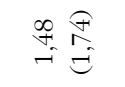 & $\begin{array}{l}\hat{\tilde{O}} \\
\hat{\tilde{o}} \\
\hat{\theta}\end{array}$ & 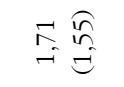 & $\underset{\approx}{\stackrel{\overbrace{}}{\rightleftarrows}}$ & $\begin{array}{l}\hat{\sigma} \\
\text { i } \\
i \\
i\end{array}$ \\
\hline ฐ & & 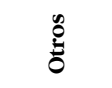 & $\begin{array}{l}8 \text { ह̂ } \\
\text { है } \\
0\end{array}$ & $\begin{array}{ll}1 & \hat{\theta} \\
0 & 0 \\
0 & 0\end{array}$ & $\begin{array}{ll}\infty & \hat{F} \\
0 & 0\end{array}$ & $\begin{array}{l}\approx \\
\approx \\
0 \\
\tilde{e}\end{array}$ & 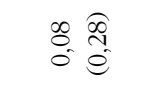 \\
\hline . & & $=\frac{\mathscr{Q}}{g}$ & $\begin{array}{l}n \text { त् } \\
\text { ô } \\
0 \text { है }\end{array}$ & 0 & 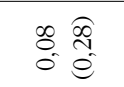 & $\begin{array}{l}m \\
\tilde{0} \\
\tilde{e}\end{array}$ & 0 \\
\hline$\frac{5}{8}$ & & $\dot{4}$ & $\begin{array}{l}2 \text { त̂ } \\
0 \text { है }\end{array}$ & 0 & 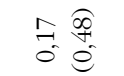 & 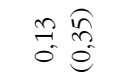 & $\begin{array}{ll}\infty & 0 \\
0 \\
0 & 0 \\
0 & 0\end{array}$ \\
\hline & & 安䓛 & 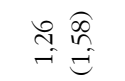 & $\begin{array}{l}\text { if } \widehat{\hat{b}} \\
0 \\
0\end{array}$ & $\begin{array}{l}\infty \\
\stackrel{\infty}{\mathbb{N}} \\
\stackrel{\sim}{=}\end{array}$ & 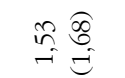 & $\begin{array}{l}\Re \underset{n}{\tilde{n}} \\
\hat{i} \underset{d}{d}\end{array}$ \\
\hline : & \multirow{8}{*}{ 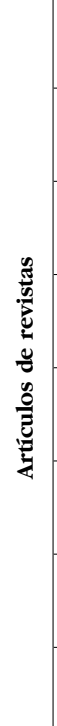 } & 롱 & $\begin{array}{l}\text { 요 } \widehat{8} \\
\text { in ti }\end{array}$ & $\begin{array}{l}1 \\
\stackrel{+}{*} \stackrel{d}{d}\end{array}$ & 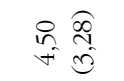 & 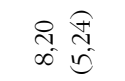 & 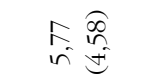 \\
\hline & & $\stackrel{0}{0}$ & 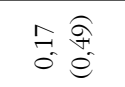 & $\begin{array}{cc}\vec{r} & \hat{\sigma} \\
0 & \stackrel{n}{0}\end{array}$ & 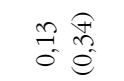 & 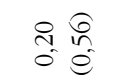 & 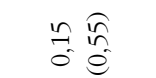 \\
\hline ป & & 商 & 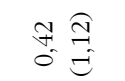 & $\begin{array}{l}\vec{n} \\
\stackrel{\hat{T}}{0} \hat{e}\end{array}$ & 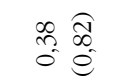 & 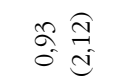 & 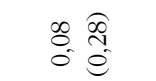 \\
\hline$\sqrt[3]{5}$ & & $\dot{\mathscr{E}}$ & 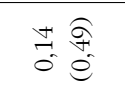 & 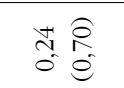 & 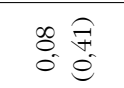 & \begin{tabular}{ll}
1 & $\hat{\sigma}$ \\
0 & \multirow{1}{*}{} \\
0 & 0
\end{tabular} & $\begin{array}{ll}\infty & \widehat{0} \\
0 & \stackrel{1}{1} \\
0 & 0\end{array}$ \\
\hline so & & 过䓪 & $\exists \approx$ & 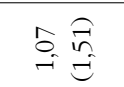 & 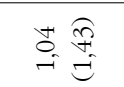 & 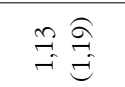 & $\overrightarrow{\vec{r}} \underset{\vec{d}}{\stackrel{f}{d}}$ \\
\hline$\frac{2}{8}$ & & 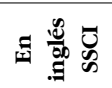 & $\begin{array}{ll}n & \widehat{2} \\
0 & \stackrel{0}{e}\end{array}$ & $\begin{array}{ll}1 & 0 \\
0 & 1 \\
0 & 0\end{array}$ & $\begin{array}{ll}* & \widehat{d} \\
0 & 0 \\
0 & 0\end{array}$ & 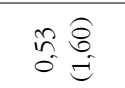 & $\begin{array}{ll}\infty & \widehat{d} \\
0 & \mathbb{1} \\
0 & e\end{array}$ \\
\hline 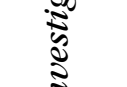 & & 雷兽 & $\stackrel{\substack{n \\
=}}{\approx}=$ & 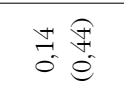 & 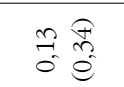 & 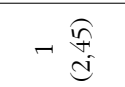 & 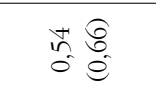 \\
\hline$\tilde{s}$ & & $\dot{4}$ & $\begin{array}{l}\hat{i} \\
\vec{i} \\
\vec{i} \\
\tilde{d}\end{array}$ & $\begin{array}{l}\mathfrak{N} \\
\hat{i} \\
\stackrel{d}{d}\end{array}$ & 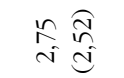 & 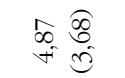 & $\begin{array}{l}\vec{b} \underset{\tilde{n}}{\tilde{d}} \\
\vec{i}\end{array}$ \\
\hline 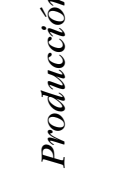 & & 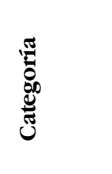 & 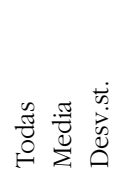 & 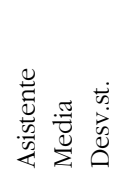 & 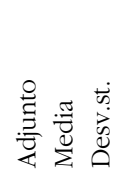 & 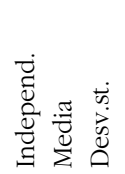 & 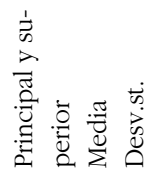 \\
\hline
\end{tabular}




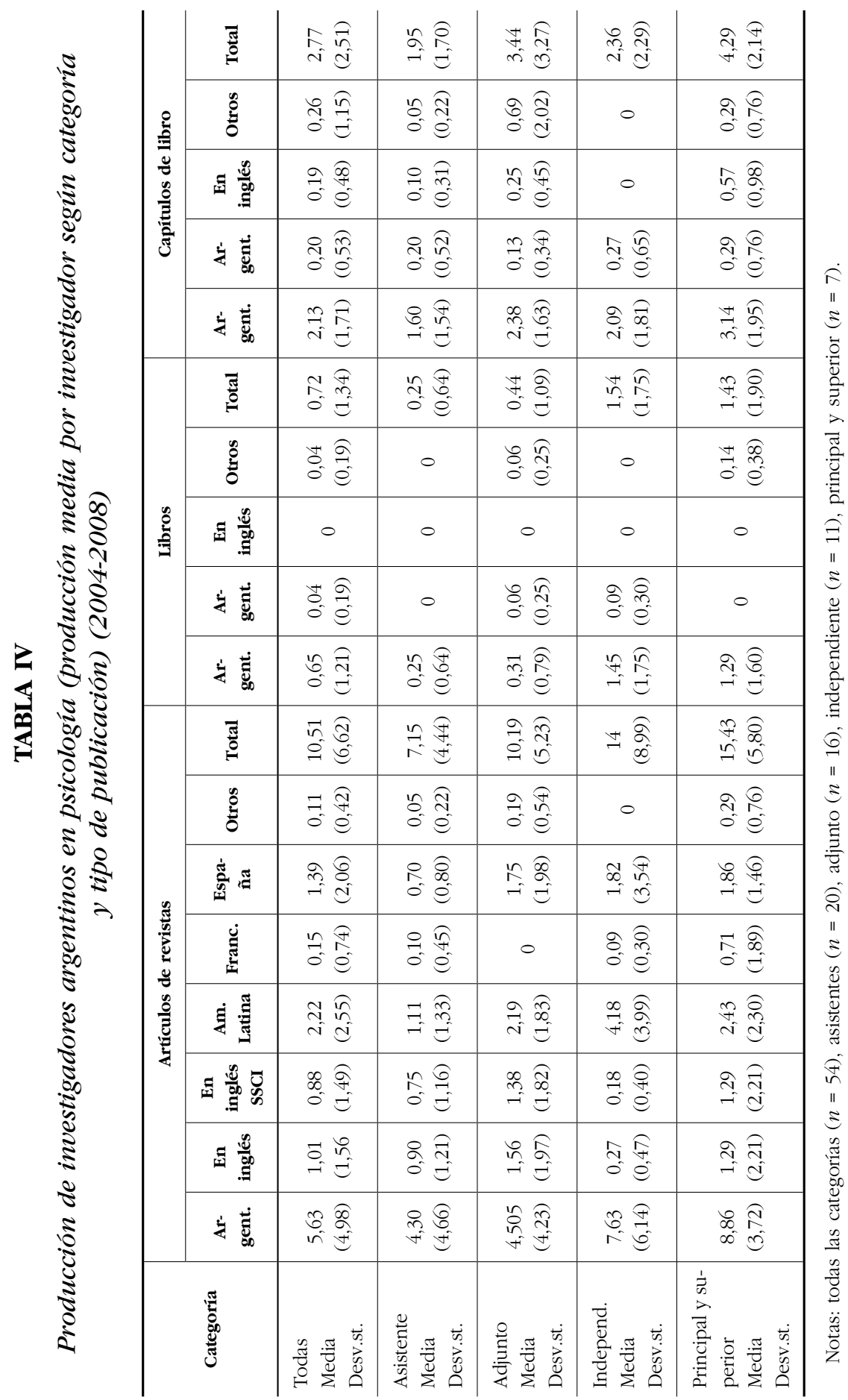


(aunque los investigadores de la categoría consolidada principal y superior tienen un número de libros levemente inferior al de la categoría inmediata anterior).

En la tabla V, se presentan algunos indicadores comparativos de categorías seleccionadas en las cuatro disciplinas. En general, los valores son muy similares para artículos en publicaciones locales, total de artículos y total de libros, excepto en el caso de la psicología, que muestra un patrón distinto de publicaciones con un número de artículos que duplica o casi duplica al de las otras disciplinas, y un número menor de libros publicados. En materia de artículos en inglés, no obstante, la economía supera a la psicología $(1,22$ contra 1,01), aunque los psicólogos tienen un número de artículos en inglés indexados en el SSCI ligeramente superior al de los economistas $(0,88$ contra 0,70$)$. La economía es la disciplina con un mayor grado de internacionalización (51,40\%) seguido por la sociología, la psicología y la ciencia política, aunque en valores absolutos la mayor productividad de artículos a nivel internacional corresponde a los psicólogos.

Por otra parte, es de destacar el carácter regional (local más resto de América Latina) de la producción de la sociología con $82,68 \%$ del total y la ciencia política $(80,22 \%)$. En cambio, la economía, si bien tiene carácter predominantemente regional, presenta un porcentaje menor (68\%). Específicamente, la economía muestra una mayor orientación hacia el mundo anglosajón y mayor visibilidad dentro del conjunto de publicaciones de más impacto internacional, mientras que la sociología y la ciencia política tienen un carácter más local y regional. La psicología se ubica en una posición intermedia en términos porcentuales, aunque en valores absolutos de producción de artículos en inglés tiene una presencia similar a la economía.

Por último, se han comparado los valores de las medias de producción por investigador en las distintas disciplinas entre sí a través del test de Mann-Whitney, indicándose en la tabla las diferencias que presentan significatividad estadística. Así, por ejemplo, se concluye que la media de artículos publicados en inglés y en revistas en dicho idioma indexadas en el SSCI es estadísticamente diferente entre economía y sociología y entre economía y ciencia política, pero no así entre economía y psicología. En general, salvo este último caso, los patrones de producción de los investigadores en psicología son significativamente diferentes de los del resto de las disciplinas. Además, no se observa en las categorías de la tabla $\mathrm{V}$ ninguna diferencia significativa entre la productividad media de los sociólogos y los investigadores en ciencia política, lo cual revela que en el ámbito argentino resultan disciplinas afines en sus patrones de publicación.

Otra forma de acercarse al fenómeno de la internacionalización de la producción científica es analizando la formación recibida por los investigadores. En tal sentido, cabría esperar que investigadores formados en el extranjero tengan mayor propensión a publicar en el circuito dentro del cual se formaron. La vinculación, y posiblemente causalidad, entre formación obtenida en el extranjero y productividad a nivel de publicaciones internacionales ya había sido notada para el caso de los investigadores en economía por García et al. (1999). Del mismo modo, García Romero (2002) demuestra la importancia de la formación predoctoral para 


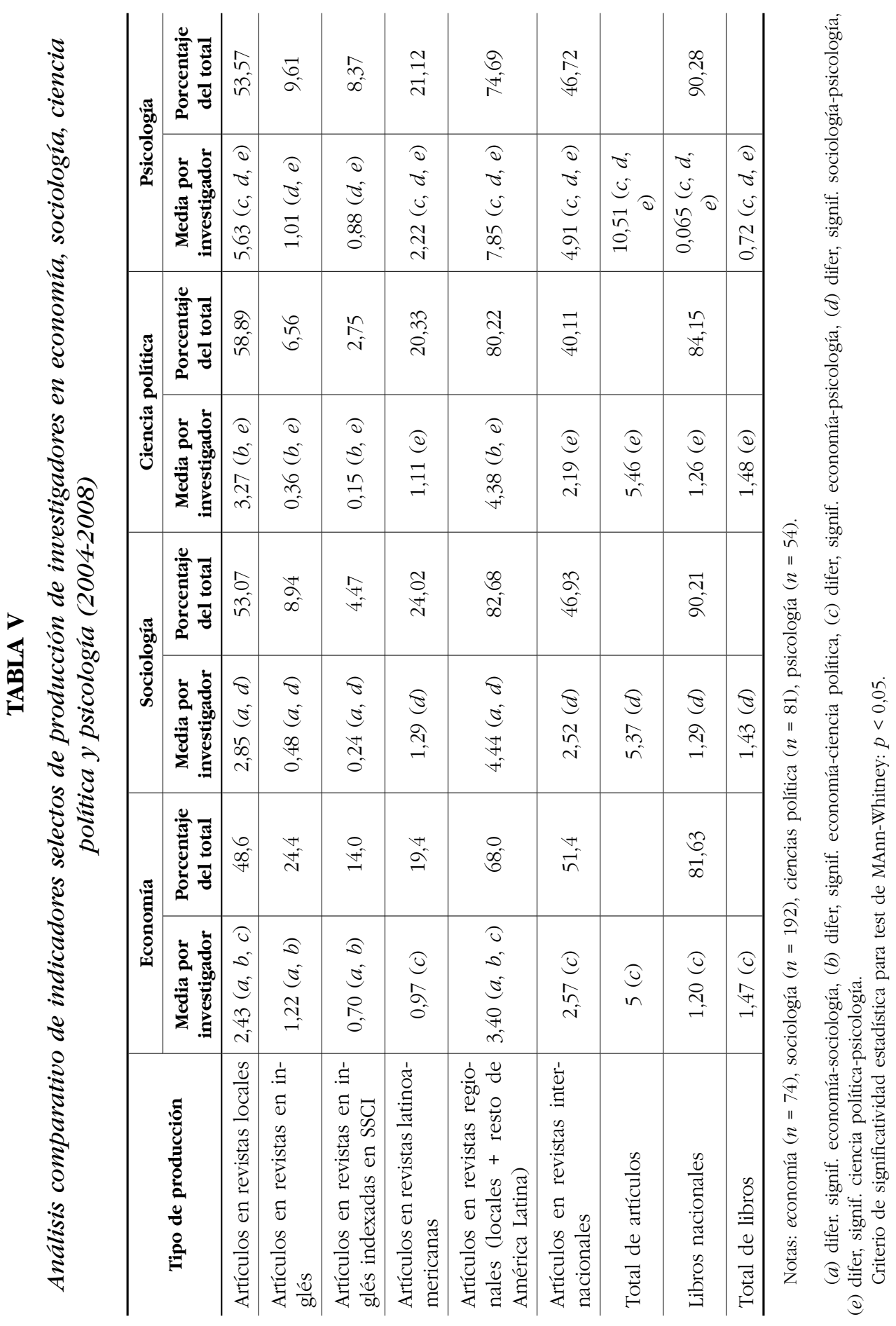


la productividad posterior. No obstante, no hay estudios que vinculen la formación doctoral con la orientación de las publicaciones a distintos circuitos internacionales. Para analizar esta relación, se ha realizado un análisis multivariado tomando como variables independientes los lugares de obtención del doctorado y considerando como variables dependientes distintas categorías de publicaciones, básicamente artículos y libros locales (por su escaso número no se consideró la publicación de libros en otros países). Los resultados de la regresión se detallan en la tabla VI.

\section{TABLA VI}

Regresión binomial negativa de la productividad de investigadores argentinos en economía, sociología, psicología y ciencias políticas (2004-2008)

\begin{tabular}{|c|c|c|c|c|c|c|c|}
\hline $\begin{array}{l}\text { Variables indepen- } \\
\text { dientes }\end{array}$ & $\begin{array}{l}\text { Arts. rev. } \\
\text { locales }\end{array}$ & $\begin{array}{c}\text { Arts. rev. } \\
\text { Am. } \\
\text { Latina }\end{array}$ & $\begin{array}{l}\text { Arts. rev. } \\
\text { en inglés }\end{array}$ & $\begin{array}{l}\text { Arts. rev. } \\
\text { en inglés } \\
\text { SSCI }\end{array}$ & $\begin{array}{l}\text { Arts. rev. } \\
\text { Francés }\end{array}$ & $\begin{array}{l}\text { Arts. rev. } \\
\text { España }\end{array}$ & $\begin{array}{c}\text { Libros } \\
\text { naciona- } \\
\text { les }\end{array}$ \\
\hline Constante & $\begin{array}{c}1,332 \\
(0,061)\end{array}$ & $\begin{array}{c}0,307 \\
(0,088)\end{array}$ & $\begin{array}{l}-0,635 \\
(0,137)\end{array}$ & $\begin{array}{l}-1,117 \\
(0,175)\end{array}$ & $\begin{array}{l}-2,013 \\
(0,202)\end{array}$ & $\begin{array}{l}-0,880 \\
(0,140)\end{array}$ & $\begin{array}{c}0,125 \\
(0,087)\end{array}$ \\
\hline Doct. USA & $\begin{array}{c}-0,582^{* * * *} \\
(0,184)\end{array}$ & $\begin{array}{c}-0,537^{* *} \\
(0,269)\end{array}$ & $\begin{array}{l}1,040^{* * *} \\
(0,322)\end{array}$ & $\begin{array}{l}1,025^{* *} \\
(0,410)\end{array}$ & $\begin{array}{l}-0,821 \\
(0,763)\end{array}$ & $\begin{array}{c}-1,260 * * \\
(0,569)\end{array}$ & $\begin{array}{l}-0,041 \\
(0,239)\end{array}$ \\
\hline Doct. Reino Unido & $\begin{array}{c}-0,890^{\text {***** }} \\
(0,264)\end{array}$ & $\begin{array}{l}-0,713^{*} \\
(0,378)\end{array}$ & $\begin{array}{l}1,002^{* *} \\
(0,425)\end{array}$ & $\begin{array}{l}0,935^{*} \\
(0,545)\end{array}$ & $\begin{array}{l}-0,185 \\
(0,787)\end{array}$ & $\begin{array}{l}-1,317^{*} \\
(0,788)\end{array}$ & $\begin{array}{c}-1918^{* * * * *} \\
(0,619)\end{array}$ \\
\hline Doct. Francia & $\begin{array}{c}-0,602^{* * * *} \\
(0,205)\end{array}$ & $\begin{array}{l}-0,558^{*} \\
(0,300)\end{array}$ & $\begin{array}{c}0,230 \\
(0,396)\end{array}$ & $\begin{array}{c}0,124 \\
(0,513)\end{array}$ & $\begin{array}{l}2,118^{* * *} \\
(0,356)\end{array}$ & $\begin{array}{l}-0,018 \\
(0,422)\end{array}$ & $\begin{array}{c}0,340 \\
(0,243)\end{array}$ \\
\hline Doct. España & $\begin{array}{c}0,162 \\
(0,197)\end{array}$ & $\begin{array}{c}0,473^{*} \\
(0,267)\end{array}$ & $\begin{array}{l}-0,377 \\
(0,495)\end{array}$ & $\begin{array}{l}-0,875 \\
(0,726)\end{array}$ & $\begin{array}{l}-0,385 \\
(0,778)\end{array}$ & $\begin{array}{c}1,778^{* * * *} \\
(0,349)\end{array}$ & $\begin{array}{c}0,185 \\
(0,275)\end{array}$ \\
\hline Doct. Am. Latina & $\begin{array}{c}-0,416^{* * *} \\
(0,194)\end{array}$ & $\begin{array}{c}0,637^{* * *} \\
(0,235)\end{array}$ & $\begin{array}{l}-0,500 \\
(0,457)\end{array}$ & $\begin{array}{c}-1,522^{*} \\
(0,812)\end{array}$ & $\begin{array}{c}0,472 \\
(0,508)\end{array}$ & $\begin{array}{c}0,033 \\
(0,410)\end{array}$ & $\begin{array}{c}0,098 \\
(0,252)\end{array}$ \\
\hline Doct. otros países & $\begin{array}{l}-0,273 \\
(0,237)\end{array}$ & $\begin{array}{l}-0,575 \\
(0,373)\end{array}$ & $\begin{array}{c}0,692^{*} \\
(0,453)\end{array}$ & $\begin{array}{c}0,769 \\
(0,570)\end{array}$ & $\begin{array}{l}-0,127 \\
(0,790)\end{array}$ & $\begin{array}{c}0,532 \\
(0,459)\end{array}$ & $\begin{array}{l}-0,014 \\
(0,324)\end{array}$ \\
\hline Log. likelihood & $-823,19$ & $-567,20$ & $-385,93$ & $-273,84$ & $-171,77$ & $-314,98$ & $-527,04$ \\
\hline Parámetro alpha & 0,55 & 0,94 & 2,22 & 3,59 & 1,41 & 1,83 & 0,76 \\
\hline
\end{tabular}

Notas: $n=363,{ }^{*} \mathrm{p}<0,10,{ }^{* *} \mathrm{p}<0,05,{ }^{* * *} \mathrm{p}<0,01,{ }^{* * * *} \mathrm{p}<0,001$, desvío standard entre paréntesis.

Para dicho análisis, la muestra es menor a la considerada en el análisis de productividad porque no fue posible obtener datos sobre la universidad en que completaron su formación doctoral algunos investigadores (véase la distribución en la tabla VII). Además, en los casos en que el doctorado fue obtenido en cotutela (típicamente entre una universidad francesa y la Universidad de Buenos Aires), se lo considera como local. 
TABLA VII

Investigadores por lugar de formación doctoral

\begin{tabular}{|l|c|c|}
\hline \multicolumn{1}{|c|}{ Lugar de formación doctoral } & Número & Porcentaje \\
\hline Doctorado en USA & 34 & 9,37 \\
\hline Doctorado en el Reino Unido & 18 & 4,96 \\
\hline Doctorado en Francia & 27 & 7,44 \\
\hline Doctorado en España & 22 & 6,06 \\
\hline Doctorado en países de América Latina $\left(^{*}\right)$ & 28 & 7,71 \\
\hline Doctorado en otros países $\left(^{* *}\right)$ & 17 & 4,68 \\
\hline Doctorado local & 217 & 59,68 \\
\hline Total & 363 & 100 \\
\hline
\end{tabular}

Notas: $n=363$.

(*) Brasil (17), México (10), Venezuela (1).

(**) Holanda (6), Alemania (4), Italia (3), Bélgica (2), Suecia (1), Canadá (1).

En este análisis, la categoría base es el doctorado local, por lo cual los coeficientes deben interpretarse como la medida en que obtener un doctorado en una de las localizaciones mencionadas aumenta o disminuye la probabilidad de efectuar determinadas publicaciones con relación a los investigadores que tienen un doctorado local. Así, tener un doctorado en USA, el Reino Unido, Francia o países latinoamericanos reduce la productividad en publicaciones locales en forma estadísticamente significativa. Respecto a las publicaciones en revistas de países de América Latina, se aprecia que, consistentemente con nuestra expectativa, poseer un doctorado en países de América Latina tiene un efecto positivo y estadísticamente significativo sobre la productividad en tales revistas. Tener un doctorado en España también afecta positivamente la productividad en revistas latinoamericanas, mientras que la formación doctoral en USA, Francia y el Reino Unido tiene también un efecto significativo pero negativo. En el caso de las revistas en inglés, sólo tienen efectos estadísticamente significativos y positivos el doctorado en USA, Reino Unido y otros países. Los resultados son similares cuando se trata de publicaciones en inglés indexadas en el SSCI. Los efectos estadísticamente significativos positivos respecto a la categoría base corresponden a doctorados en USA y el Reino Unido, en tanto que la formación doctoral en un país latinoamericano tiene un efecto signficativo pero negativo. En el caso de las revistas francesas, el resultado es esperable ya que sólo tiene efecto significativo y positivo el haber obtenido un doctorado en Francia. En lo atinente a las publicaciones en revistas españolas, los resultados con significatividad estadística corresponden a doctorados en España (efecto positivo) y en USA y el Reino Unido 
(efecto negativo). Respecto a las publicaciones de libros en Argentina, la única variable con efecto estadísticamente significativo y negativo es la obtención de un doctorado en el Reino Unido. En general, estos resultados sugieren que existe un interés por parte de quienes se formaron en distintos países en seguir participando en los circuitos de publicaciones de los mismos. Posiblemente, esto obedezca tanto a razones de índole lingüística como al establecimiento de redes de colaboración con investigadores de su lugar de formación doctoral.

\section{Conclusión}

El presente trabajo avanza sobre la literatura que alerta sobre el bajo nivel de presencia en las principales bases de datos científicas de investigadores en ciencias sociales de países en desarrollo, al cuantificar su productividad, tanto en revistas locales como en revistas internacionales, así como en otros tipos de medios de difusión de investigaciones. A partir del caso de la República Argentina, evaluado a través de la recopilación de datos de publicaciones de un conjunto de académicos, se demuestra y cuantifica la preponderancia de publicaciones en revistas científicas locales. Los resultados indican, además, que esto es dependiente del tipo de disciplina considerada, ya que dentro de las ciencias sociales hay disciplinas que tienen un mayor perfil de apertura al circuito de publicaciones anglosajón, siendo pertinente en este sentido el ejemplo de la economía. La sociología y la ciencia política, por el contrario, manifiestan un mayor carácter localista o, más específicamente, regionalista. La psicología, por su parte, evidencia un patrón de publicaciones distinto a las anteriores, con una mayor producción de artículos en revistas científicas. Esta disciplina tiene en términos porcentuales una amplia participación regional, pero también presencia en publicaciones científicas en inglés.

Por último, otro hallazgo importante es la existencia de un nexo entre la elección geográfica de las publicaciones por parte de los investigadores y su lugar de formación doctoral, lo cual no es algo exclusivo de quienes publican en revistas en inglés sino que se verifica con publicaciones efectuadas en otros idiomas.

Si bien no son muchos los estudios empíricos que posibilitan el análisis comparativo, algunos trabajos sugerirían que la tendencia a publicar en el ámbito latinoamericano es común entre científicos de la región, como demuestran Vessuri y otros (2001) en el caso de Venezuela. Además, y estrictamente en el caso de la economía, Faria (2000) encuentra una baja participación de economistas brasileños en revistas internacionales. No obstante, y especialmente teniendo en cuenta la importancia que últimamente se asigna a la visibilidad internacional de las ciencias sociales de países en desarrollo (Díaz Escoto y otros, 2010; Dwyer, 2009), serían necesarios nuevos análisis similares de otros países en desarrollo para evaluar el grado de importancia de la literatura orientada a la difusión local frente a aquella orientada a una audiencia internacional. 


\section{Bibliografía}

Archambault, E.; Vignola-Gagne, E.; Côté, G, Larivière, V., y Gingras, Y. (2006): Benchmarking scientific output in the social sciences and humanities: The limits of existing databases. Scientometrics, vol. 68 (3), 329-342.

Archambault, E., y Larivière, V. (2010): The limits of bibliometrics for the analysis of the social sciences and humanities literature. En: International Social Science Council, World Social Sciences Report: Knowledge Divides, UNESCO, París.

CAYCIT - Centro Argentino de Divulgación Científica y Tecnológica (2005): Producción científica argentina en Science Citation Index 2004. http://www.caicyt.gov.ar/basesde-datos-e-indicadores/documentos-1/produccionconicet_2004.pdf (con acceso 15.04.2011).

CONICET - Consejo Nacional de Investigaciones Científicas y Técnicas (2006): 50 años del CONICET y la historia de la ciencia en Argentina. CONICET, Buenos Aires.

Díaz Escoto, A.; Navarro, E., y Ramírez Godoy, M. E. (2010): Contribución científica de México a la psicología entre los años 1995-2008 con base en el Social Sciences Citation Index de ISI Web of Knowledge, Biblioteca Universitaria, vol. 13 (1), 16-35.

Dierz, J. S.; Chompolov, I.;Bozeman B.; Lane, E., y Park, J. (2003): Using the curriculum vitae to study the career paths of scientists and engineers: An exploratory assessment. Scientometrics, vol. 49 (3), 419-442.

Dwyer, T. (2009): On the Internationalization of Brazilian Academic Sociology. En: Burawoy, M.; Chang, M.; Hsieh, M. F. (eds.), Facing An Unequal World: Challenges for a global sociology, vol. 1, Academia Sinica, Taipei: Taiwan.

Faria, J. R. (2000): The research output of academic economists in Brazil. Economia Aplicada, vol. 4 (1), 95-113.

Gaillard, J. (1992): Use of publication lists to study scientific production and strategies of scientists in developing countries. Scientometrics, vol. 23(1), 57-73.

García, P.; Montañés, A., y Sanz, E. (1999): La investigación en economía en España: mercado nacional ¿versus? mercado internacional. Revista de Economía Aplicada, vol. 7 (20), 167-187.

García Romero A. (2002): Ensayos sobre Evaluación de la Investigación: Efectos de los Programas Predoctorales y Postdoctorales, Tesis doctoral, Universidad Autónoma de Madrid.

Giannoni, D. (2008): Medical writing at the periphery: The case of Italian journal editorials. Journal of English for Academic Purposes, vol. 7 (2), 97-107.

Hicks, D. (2004): The four literatures of social science. En: Moed, H.; Glänzel, W.; Schmoch, U. (eds.), Handbook of quantitative science and technology research. Kluwer, Dordrecht.

Jiménez Contreras, E. (2002): Las revistas científicas: el centro y la periferia. Revista Española de Documentación Científica, vol. 15 (2), 174-82.

Kahn, M. (2010): Measure for measure: quantifying the social sciences. En International Social Science Council, World Social Sciences Report: Knowledge Divides. UNESCO, París.

Kishida, K., y Matsui, S. (1997): International publication patterns in social sciences: a quantitative analysis of the IBSS files. Scientometrics, vol 40 (2), 277-298. 
Luna-Morales, M. E., y Collazo-Reyes, F. (2007): Análisis histórico bibliométrico de las revistas latinoamericanas y caribeñas en los índices de la ciencia internacional: 19612005. Revista Española de Documentación Científica, vol. 30 (4), 523-543.

Narvaez-Berthelemot, N., y Russell, J. M. (2001): World distribution of social science journals: A view from the periphery. Scientometrics, vol. 51 (1), 223-239.

Ministerio de Ciencia, Tecnología e Innovación Productiva de la República Argentina (2009): Indicadores de ciencia y tecnología argentina, 2008, Buenos Aires.

Molteni, V., y Zulueta, M. A. (2002): Análisis de la visibilidad internacional de la producción científica argentina en las bases de datos Social Sciences Citation Index y Arts and Humanities Citation Index en la década de 1990-2000: estudio bibliométrico. Revista Española de Documentación Científica, vol. 25 (4), 455-465.

Morillo, F.; Fernández, M., y Gómez, I. (1999): Evolution of Spanish journals in the international scene. Research Evaluation, vol. 8 (2), 71-76.

Russell, J., y Ainsworth, S. (2010): Social science research in the Latin American and the Caribbean regions in comparison with China and India. En International Social Science Council, World Social Sciences Report: Knowledge Divides. UNESCO, París.

Sancho, R. (1992): Misjudgments and shortcomings in the measurement of scientific activities in less developed countries. Scientometrics, vol 23 (1), 221-233.

Sanz, E.; Castro, F.; Povedano, E.; Hernández, A.; Martín, C.; Morillo-Velarde, J.; GarcíaZorita, C.; de la Nuez, J., y Fuentes, M. (2002): Creación de un índice de citas de revistas españolas de humanidades para el estudio de la actividad investigadora de los científicos de estas disciplinas. Revista Española de Documentación Científica, vol. 25 (4), 443-454.

Sanz Casado, E., y Conforti, N. (2005): Análisis de la actividad científica de la Facultad de Humanidades de la Universidad de Mar del Plata, durante el período 1998-2001. Revista Española de Documentación Científica, vol. 28 (2), 196-205.

Shenhav, Y., y Kamens, D. (1991): The "costs" of institutional isomorphism in non-Western countries. Social Studies of Science, vol 21 (3), 427-545.

Sztompka, P. (2009): One Sociology or Many? En. S. Patel (ed.), The ISA Handbook of Diverse Sociological Traditions, Sage, Los Angeles.

Van Leeuwen, T. N.; Moed, H. F.; Tijssen, R. J.; Visser, M. S., y Van Raan, A. (2001): Language biases in the coverage of the Science Citation Index and its consequences for international comparisons of national research performance. Scientometrics, vol. 51 (1), 335-346.

Vessuri, H. (1995): Recent strategies for adding value to scientific journals in Latin America. Scientometrics, vol 34 (1), 139-161.

Vessuri, H.; Martínez Larrechea E., y Estévez, B. (2001): Los científicos sociales en Venezuela. Perfil bibliográfico e implicaciones de política, Cuadernos del Cendes vol. 18 (48), 89-121.

Wainerman, C. (1999): El rey está desnudo: acerca de la formación de postgrado en ciencias sociales. Boletín Informativo - Consejo de Profesionales en Sociología, vol. 1 (5), 4-5. 